\title{
Auditory novelty oddball allows reliable distinction of top-down and bottom-up processes of attention
}

\author{
S. Debener ${ }^{\mathrm{a}, *}$, C. Kranczioch ${ }^{\mathrm{a}}$, C.S. Herrmann ${ }^{\mathrm{b}}$, A.K. Engel ${ }^{\mathrm{a}}$ \\ anstitute of Medicine, Research Center Jülich, Jülich D-52425, Germany \\ ${ }^{\mathrm{b}}$ Max Planck Institute of Cognitive Neuroscience, Leipzig, Germany
}

Received 11 April 2002; received in revised form 28 June 2002; accepted 1 July 2002

\begin{abstract}
An auditory novelty-oddball task, which is known to evoke a P3 event-related potential (ERP) in a target condition and a novelty-P3 ERP in response to task-irrelevant unique environmental sounds, was repeatedly applied to healthy participants $(n=14)$ on two separate recording sessions, 7 days apart. Both target-P3 and novelty-P3 were internally consistent and test-retest reliable. Interestingly, novelty-P3 amplitude declined from the first to the second half of each recording session, whereas no systematic alteration between both sessions occurred. The target-P3 showed the opposite pattern, i.e. a reduced amplitude from the first to the second session, but no systematic change within each session. These findings suggest that novelty-P3 amplitude changes reflect habituation, whereas target-P3 session effects may indicate the adjusted amount of processing resources invested into the task. In general, the results support the interpretation of the novelty-P3 as indicating automatic, bottom-up related aspects of attention, whereas the target-P3, in the present paradigm, seems to reflect voluntary, top-down related aspects of attention.
\end{abstract}

(c) 2002 Elsevier Science B.V. All rights reserved.

Keywords: Target-P3; Novelty-P3; Habituation; Reliability; Attention; Top-down; Bottom-up

\section{Introduction}

The experience of deviance is a fundamental biological mechanism, since the discrimination of novel from familiar events enables the organism to appropriately react to sudden changes in the environment. Novel or unpredicted stimuli involuntarily capture attention and evoke an orienting

\footnotetext{
*Corresponding author. Tel.: +49-2461-616184; fax: +492461-612820.

E-mail address: s.debener@fz-juelich.de (S. Debener).
}

response (Sokolov, 1963). This process has been described as the stimulus-driven, or bottom-up aspect of attention (e.g. Humphreys et al., 1999). The novelty-P3 event-related potential (ERP) component, which is a centrally distributed positive deflection with a latency of approximately $300 \mathrm{~ms}$, has been characterized as a physiological correlate of such responses to novelty (for review, see Friedman et al., 2001).

Since its first description, the novelty-P3 has been related to orienting and habituation (Cour- 
chesne et al., 1975). This view is bolstered by the fact that the novelty-P3 amplitude decreases during successive experimental blocks (e.g. Friedman and Simpson, 1994). Furthermore, its amplitude is reduced for the repeated presentation of (formerly novel) stimuli within the same stimulus sequence (Cycowicz et al., 1996). This and further evidence suggests that the novelty-P3 reflects involuntary and automatic attentional processing, a mechanism that is possibly associated with prefrontal brain structures (Daffner et al., 2000; Knight, 1984).

The presentation of novel stimuli is commonly included in an active oddball paradigm, where the subject's task is to respond to a rarely presented target stimulus that is embedded in a train of a frequently presented standard stimulus. The rare, task-relevant stimulus evokes a parietally distributed P300 (which is in the following referred to as target-P3; see Donchin et al., 1997). In comparison to the target-P3, the novelty-P3 occurs typically with a shorter latency and shows a more frontal distribution (Fabiani and Friedman, 1995; Friedman et al., 2001; Herrmann and Knight, 2001). Hence, the novelty-P3 can be regarded as a unique entity distinct from the target-P3 (Donchin et al., 1997).

Interestingly, numerous studies have explored 'habituation' of the target-P3 in the oddball paradigm (e.g. Carrillo-de-la-Pena and Garcia-Larrea 1999; Ivey and Schmidt, 1993; Kinoshita et al., 1996; Lammers and Badia, 1989; Pan et al., 2000; Polich and McIsaac, 1994; Ravden and Polich, 1998; Romero and Polich, 1996), but, however, yielded heterogenous results. If target-P3 amplitude reductions were observed, these could be attributed to several experimental variables, among them the number of target stimuli, length of interblock intervals, and length of intertarget intervals (Carrillo-de-la-Pena and Garcia-Larrea, 1999; Ravden and Polich, 1998). From these effects, it has been concluded that reduction of the target-P3 at least partly reflects a change in the amount of attentional resources invested in a task (e.g. Polich and McIsaac, 1994; Lammers and Badia, 1989). Accordingly, many authors interpret the target-P3 as reflecting the degree of cognitive involvement and top-down driven aspects of attention in the task. Given this is a correct interpretation, the term 'habituation' as a descriptor of target-P3 amplitude reduction is misleading.

In the present study, repetition effects on novelty-P3 and target-P3 were explored by applying an auditory novelty oddball paradigm on two separate sessions, 7 days apart. Due to its familiarity, a simple oddball task should require less effort to be performed well in the second session. If the target-P3 indeed reflects the amount of resources required for task performance, its amplitude should be reduced in the second recording session as a result of previous experience with the task. The novelty-P3, on the other hand, should reflect short-term habituation effects. Therefore, a reduced novelty-P3 amplitude was predicted for the second as compared to the first half of each recording session. The repeated administration of the identical set of novel stimuli after an interval of 7 days was, however, not necessarily expected to cause a reduction of the novelty-P3 amplitude, because the novel stimuli were task-irrelevant.

Recently, co-registration of ERPs with functional magnetic resonance imaging (fMRI) was performed for the auditory novelty-oddball task, and provided further insights on the neural circuits of novelty processing (Opitz et al., 1999). Such approaches require the repeated administration of the same experimental paradigm in the same subject in order to combine the excellent temporal resolution of ERPs with the excellent spatial resolution of fMRI (e.g. Nunez and Silberstein, 2000). Whereas target-P3 is known to be relatively test-retest reliable (e.g. Kinoshita et al., 1996), little is yet known about the effects of the repeated administration of the novelty-P3 paradigm. Thus, a second aim of the present study was to explore the psychometric characteristics of target-P3 and novelty-P3. Furthermore, N100 was analyzed to address a recent report on N100 habituation (Carrillo-de-la-Pena and Garcia-Larrea, 1999).

\section{Methods}

Participants were recruited among the staff of the Institute of Medicine at the Research Center Juelich, and were required to be free of past or current psychiatric and neurological disorders. Subject ages ranged from 25 to 36 (mean 30.14; 
S.D. 4.04). From the 14 participants included, 9 were male and 12 were right-handed. Normal hearing (i.e. $<30 \mathrm{~dB}$ hearing loss between 0.125 and $8 \mathrm{kHz}$ ) was confirmed using a standard threshold detection procedure.

EEG was recorded in a dimly lit, sound-attenuated and electrically shielded chamber using a high impedance 64 channel Net Amps 200 system (Electrical Geodesics, Inc., Eugene, Oregon) with a geodesic sensor net (GSN), and a vertex reference $(\mathrm{Cz})$. Sensor impedances were maintained below $30 \mathrm{k} \Omega$ prior to data acquisition (Tucker, 1993). The EEG signal was analog filtered from 0.1 to $100 \mathrm{~Hz}$, and digitized at $500 \mathrm{~Hz}$ with 0.024 $\mu \mathrm{V}$ precision. Auditory stimuli were presented binaurally at approximately $70 \mathrm{~dB}(\mathrm{SPL})$ through foam protected air-tube earphones.

Subjects were presented with a three class auditory oddball task. Two sinus tones of 350 and 650 $\mathrm{Hz}$ (340 ms duration, $10 \mathrm{~ms}$ rise time, $30 \mathrm{~ms}$ fall time) served as frequent and target tones, respectively. Which tone served as a target or frequent was counterbalanced across subjects. Target probability was $10 \%$ and frequent probability was $80 \%$. The third class $(10 \%)$ was a set of novel stimuli established by Fabiani and Friedman (1995). From this series, a total of 72 unique environmental sounds belonging to six different categories (i.e. animal, machine, music, human, electronic, bird call) with a mean duration of $340 \mathrm{~ms}$ were used. Overall, 180 stimuli were presented for each block using a variable interstimulus interval (960-1360 $\mathrm{ms}$ ). One recording session consisted of 4 blocks, with a 1-min break between blocks. To maintain attention to the targets for consecutive blocks, 03 additional targets were included at the end of each block, but these dummy trials were excluded from ERP analysis. The subjects task was to silently count the target tones. A retest session was scheduled 7 days later at the same daytime. Apart from block order, which was reversed, the same protocol was applied for the first and second recording session. Note that each novel stimulus was presented only once within a session, but was presented for a second time at the retest after 7 days.

EEG data analysis was performed using EEProbe 3.2 (ANT, Enschede, NL) and started with linear interpolation of bad channels. Across the whole data set, $0.8 \%$ of all channels were defined as bad and had to be replaced. Data were then rereferenced to the common average to obtain a reference-free transformation. EEGs were $0.5 \mathrm{~Hz}$ high-pass filtered, and marked as artifactual whenever the standard deviation within a $200 \mathrm{~ms}$ interval exceeded $20 \mu \mathrm{V}$ on any channel. This procedure was followed by visual inspection of the continuous signal. Artifact-free trials were then epoched from -200 to $800 \mathrm{~ms}$ poststimulus onset and separately averaged for the first and second half of each condition, using a prestimulus baseline correction to $0 \mu \mathrm{V}$. This procedure resulted in 12 averages for each channel and subject (2 blocks, 3 conditions, 2 sessions), which were $25 \mathrm{~Hz}$ lowpass filtered prior to statistical analysis.

The present ERP analysis focused on N100 (mean time interval $80-130 \mathrm{~ms}$ ), novelty-P3 (230-360 ms) and target-P3 (400-580 ms). In order to avoid a loss of statistical power (Oken and Chiappa, 1986), selected electrode sites were collapsed into nine regions of interest (ROI): anterior left (E09, E12, E13, E15, E16), anterior midline (E03, E04, E07, E08), anterior right (E02, E57, E58, E61, E62), temporal left (E17, 1320, $\mathrm{E} 21, \mathrm{E} 22, \mathrm{E} 24, \mathrm{E} 25)$, central midline (Cz, E05, $\mathrm{E} 18, \mathrm{E} 30, \mathrm{E} 43, \mathrm{E} 55)$, temporal right (E47, E50, E52, E53, E54, E56), posterior left (E27, E28, E29, E32, E33), posterior midline (E34, E37, E38, $\mathrm{E} 40$ ), and posterior right (E41, E42, E45, E46, E49). For spatial correspondence between the GSN positions and the 10-10 electrode positioning system see Luu and Ferree (2000).

N100 amplitude was submitted to a five-way ANOVA with the repeated measurement factors condition (frequent, target, novel), session $(1,2)$, block (a, b), caudality (anterior, central/temporal, posterior) and laterality (left, midline, right). Target-P3 and novelty-P3 ERPs were analyzed by performing a six-way ANOVA with the factors condition (target, novel), session $(1,2)$, block (a, b), caudality (anterior, central/temporal, posterior), laterality (left, midline, right) and time (230$360 \mathrm{~ms}$, 400-580 ms). Greenhouse-Geisser correction was used where appropriate. Uncorrected degrees of freedom and corrected $P$-values are reported. 
Reliability analyses focused on internal consistency and test-retest reliability, and were restricted to ROIs where ERPs showed the maximum amplitudes, i.e. the central midline ROI for N100 and novelty-P3, and the parietal midline ROI for targetP3. Internal consistency analysis was based on odd-even split-half averages, with the odd and even trials being averaged separately. This resulted in four averages per subject and condition, which were analyzed by means of Cronbachs $\alpha$. As a second method to measure reliability, test-retest reliabilities were computed on the basis of averages from the first and second session. These reliability analyses reveal an estimate of the signalto-noise ratio of ERPs (e.g. Beauducel et al., 2000), and further provide information about the homogeneity and trait-like stability of physiological measures (e.g. Debener et al., 2000).

Behavioral data were quantified by calculating a percentage score of correctly counted target tones for each block (i.e. (counted targets minus number of targets) $\times 100 /$ number of targets), and analyzed by means of a session $\times$ block repeated measurements ANOVA.

\section{Results}

Behavioral analysis of the percentage of correctly identified targets did not reveal any significant effect for block, session, or block $\times$ session (all $P>0.30$ ). All error rates were below $2.4 \%$, indicating low difficulty of the task.

The main findings for the reliability analysis of N100, target-P3 and novelty-P3 ERPs are summarized in Table 1. Internal consistency values varied between 0.83 and 0.94 . Note that the highest value was obtained for the ERP measure with the most trials included in the average, i.e. the N100 response to frequent standard tones. However, similarly good signal-to-noise ratios were obtained for the other components and conditions analyzed. A somewhat different pattern emerged for testretest reliabilities. Both $\mathrm{N} 100\left(r_{\mathrm{tt}}>0.74\right)$ and the novelty-P3 $\left(r_{\mathrm{tt}}=0.79\right)$ test-retest reliabilities were high. Yet, a substantially lower test-retest reliability was found for the target-P3 $\left(r_{\mathrm{tt}}=0.50\right)$.

ANOVA analyses for N100 revealed that neither
Table 1

Internal consistency (Cronbachs $\alpha$ ) and test-retest reliability $\left(r_{\mathrm{tt}}\right)$ for N100, target-P3 and novelty-P3 at those ROIs where the components had maximum amplitude

\begin{tabular}{|c|c|c|c|c|}
\hline \multicolumn{3}{|c|}{ Reliability measure } & \multirow[t]{2}{*}{ Cronbachs $\alpha$} & \multirow[t]{2}{*}{ Test-retest $\left(r_{\mathrm{tt}}\right)$} \\
\hline Component & Condition & ROI & & \\
\hline \multirow[t]{3}{*}{ N100 } & Standard & $\mathrm{CM}$ & 0.94 & 0.89 \\
\hline & Target & $\mathrm{CM}$ & 0.86 & 0.74 \\
\hline & Novel & $\mathrm{CM}$ & 0.85 & 0.84 \\
\hline Novelty-P3 & Novel & $\mathrm{CM}$ & 0.91 & 0.79 \\
\hline Target-P3 & Target & $\mathrm{PM}$ & 0.83 & 0.50 \\
\hline
\end{tabular}

Note: Estimates of internal consistency are based on oddeven split-half averages in both sessions. Test-retest reliabilities are based on data averaged within each session. ROI, region of interest; $\mathrm{CM}$, central midline; $\mathrm{PM}$, posterior midline.

laterality nor caudality factors showed significant interactions with block and/or session (all $P>$ 0.20 ), and thus, they were excluded from further analyses. The consecutive condition (frequent, target, novel $) \times$ session $(1,2) \times$ block $(a, b)$ ANOVA at the central midline ROI revealed a significant condition main effect, $F_{(2,26)}=6.9, P=0.006$, with the largest N100 amplitude for the target condition $(-2.1 \mu \mathrm{V})$, followed by novels $(-1.6 \mu \mathrm{V})$ and frequents $(-1.4 \mu \mathrm{V})$. All other effects were insignificant, with only the three-way interaction condition $\times$ session $\times$ block approaching significance, $F_{(2,26)}=2.74, P=0.092$.

The six-way ANOVA for target-P3 and novelty$\mathrm{P} 3$ revealed that the laterality factor did not interact with the two factors of interest, i.e., with block, session, or both (all $P>0.20)$. Laterality was therefore excluded from further analyses. Moreover, a significant three-way interaction condition $\times$ caudality $\times$ time, $\quad F_{(2,26)}=16.64, \quad P<0.001$, indicated that in the early time interval, the maximum amplitude occurred for the novel condition at the central midline ROI. In contrast, the maximum ERP amplitude for the late time interval occurred in the target condition at parietal midline ROI, confirming the known parietal topography and condition effect for the target-P3 component. Therefore, consecutive analyses were limited to separate ANOVAs for the novelty-P3 at the central midline ROI and the target-P3 at the parietal midline ROI, which allowed to directly test the 
hypothesis of different repetition effects on novelty-P3 and target-P3.

For the novelty-P3, the two-way ANOVA with session and block as factors revealed a significant main effect block, $F_{(1,13)}=6.16, P<0.0275$, with a lower amplitude in the second as compared to the first block $(5.1$ vs. $4.6 \mu \mathrm{V})$. In this analysis, neither the session main effect nor the block $\times$ session interaction approached significance (both $F<1$ ). For the target-P3 ANOVA, amplitudes were decreased at the second session, i.e. there was a significant session effect, $F_{(1,13)}=5.42$, $P<0.0367$. Neither the block main effect $(F<1)$ nor the block $\times$ session interaction, $F_{(1,13)}=1.35$, $P>0.26$, reached significance. ERPs illustrating the main findings are depicted in Fig. 1. As can be seen, the session main effect was most pronounced at the time interval defined for target-P3 analysis. Similarly, the block main effect for novel stimuli was manifest in the time interval where the novelty-P3 occurred. In addition, Fig. 2 gives the block and session means for both novelty-P3 and target-P3 amplitude. This further demonstrates that target-P3 was primarily affected between sessions, whereas novelty-P3 varied between blocks.

\section{Discussion}

The present study confirms that target-P3 and novelty-P3 ERPs, but not the N100 component, are differently affected by repeated administration of a novelty oddball paradigm. In agreement with prior findings, the novelty-P3 shows habituation, i.e., a decrease within a recording session. The target-P3, on the other hand, was reduced only in a retest after an interval of 7 days.

An important methodological result of our study is that ERP components recorded with the GSN showed good, if not excellent, internal consistency. As recently argued, ERPs recorded with the GSN may have the disadvantage of a lower signal-tonoise ratio when compared to ERPs recorded with conventional low-impedance systems (Kayser et al., 2000). A comparison of our data with a second unpublished data set where the same paradigm (identical stimuli, same number of trials, etc.) was recorded with a NeuroScan amplifier system (Neurosoft, Inc., Sterling, VA) yielded highly similar
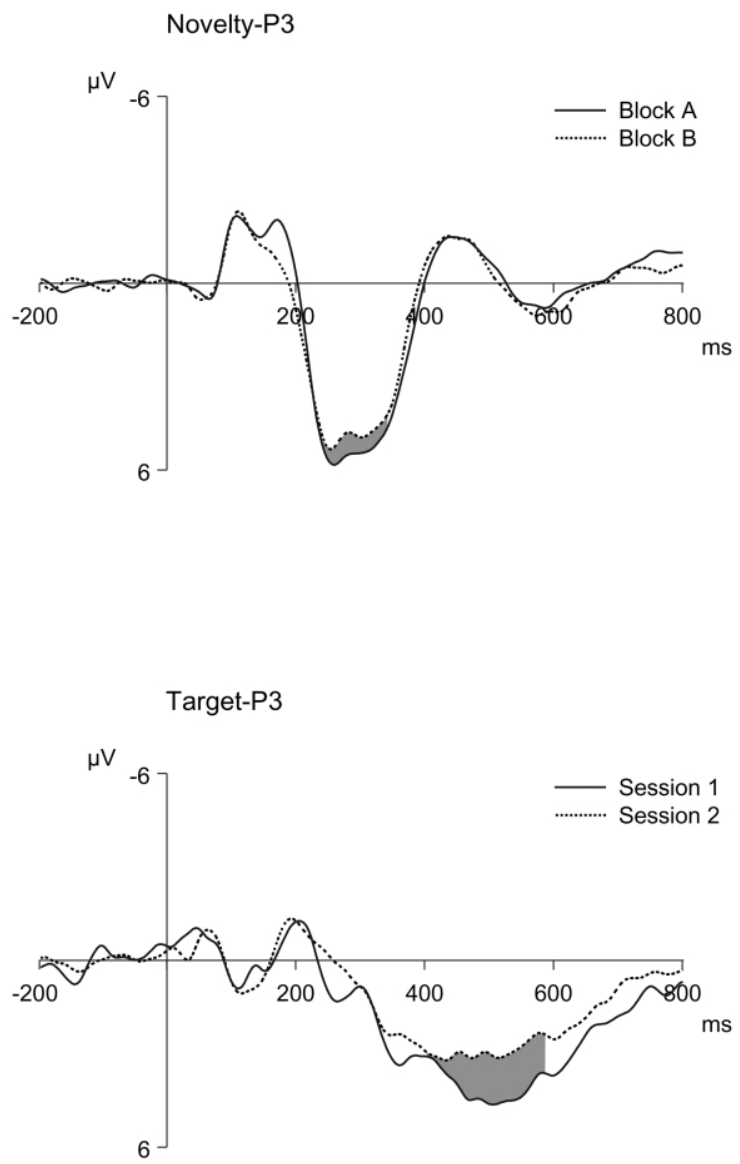

Fig. 1. Grand mean averaged $(n=14)$ ERPs evoked by novel (top) and target (bottom) stimuli in a novelty oddball paradigm. Upper half of the figure illustrates the block main effect $(P=0.0275)$ for novelty-P3 at central midline ROI. Lower part shows the session main effect $(P=0.0367)$ for target-P3 at posterior midline ROI. Grey shaded areas indicate statistically analyzed time windows.

results and, in particular, similar reliabilities. Hence, the practical implication of our data is that use of the GSN does not necessarily result in a lower signal-to-noise ratio.

At least one previous report (Carrillo-de-la-Pena and Garcia-Larrea, 1999) found a reduction of N100 amplitude across block repetition. In that study, N100 attenuation was interpreted as reflecting a process of habituation that is related to general arousal or alertness levels. In the present study, however, N100 amplitude was not signifi- 


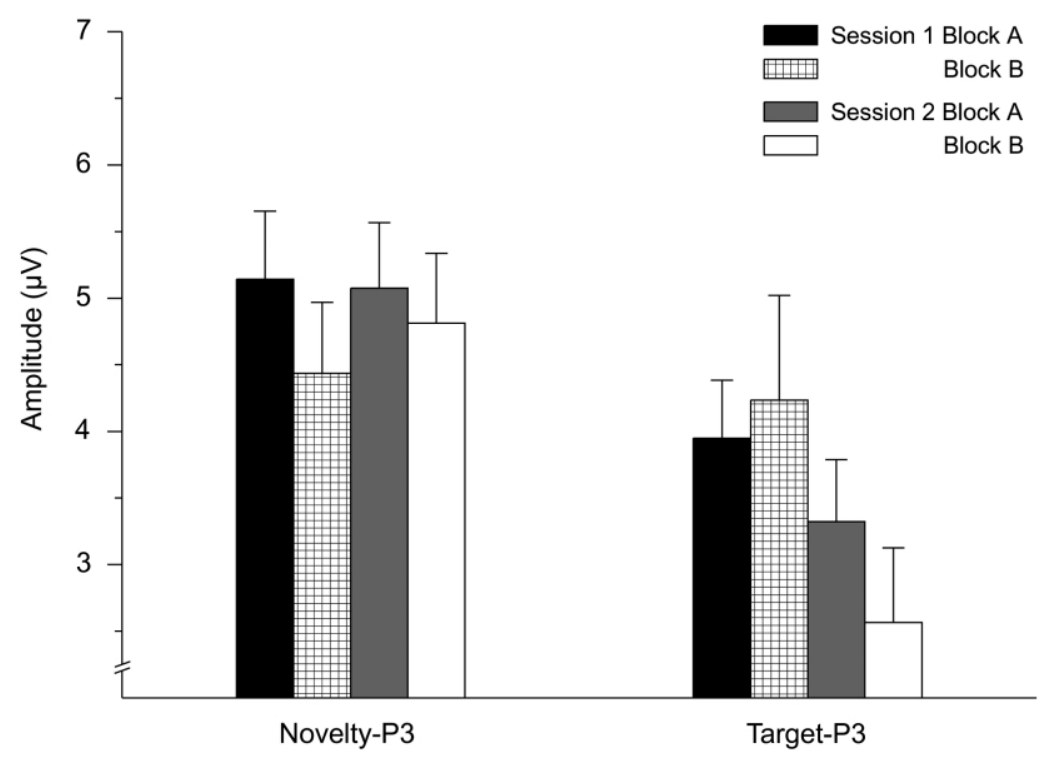

Fig. 2. Mean amplitude ( \pm S.E.M.) of novelty-P3 (left, central midline ROI) and target-P3 (right, parietal midline ROI), separately for both blocks and sessions.

cantly affected, neither by experimental blocks, nor by recording sessions or experimental conditions. Thus, it seems rather unlikely that the present results for novelty-P3 and target-P3 are related to a shift in overall arousal or alterness alone.

There has been some debate on distinct frontal and posterior aspects of the novelty-P3 ERP and its functional significance (Friedman et al., 2001). Probably related to this is the distinction between P3a and novelty-P3, which may be questioned (Simons et al., 2001). The present report was limited to the comparison of repetition effects on novelty-P3 and target-P3 mainly because a straightforward hypothesis could be derived from the literature. Speculations on a topographical shift of the novelty-P3 in response to stimulus repetition were not in the focus of the present work, and more complex experimental designs are needed to address these issues in detail.

Psychometric analyses revealed good internal consistencies of N100, target-P3 and novel-P3, with all Cronbachs $\alpha$ values being above 0.80 . This finding confirms similar signal-to-noise ratios between novel-P3 and target-P3, which is helpful for the interpretation of different test-retest reliabilities. Interestingly, the latter analysis revealed a high novelty-P3 test-retest reliability, similar to that found for the N100. For the target-P3, this analysis revealed only moderate values. However, the lower retest reliability for target-P3 is in good agreement with the interpretation of the target-P3 as reflecting voluntary attentional demands. The amount of target-P3 reduction, which may index the reduced amount of attentional resources put into task, showed relatively large variability across subjects. As compared to this, the individual response to novel stimuli can be considered a traitlike, temporally stable biological characteristic. Taken together, these findings point toward the significance of individual differences in the novelty oddball paradigm (Opitz et al., 1999). Future studies employing the EEG-fMRI co-registration approach might consider the evaluation of temporal characteristics.

Across subjects, assessment of amplitude changes between the first and second half of each recording session revealed a significant reduction of the novelty-P3. Similar to others, we interpret this finding as reflecting habituation (Cycowicz et al., 1996; Friedman et al., 2001). As they were task-irrelevant, no special action by the participants was required in response to the novel stimuli. 
Thus, the block effect observed for the novelty-P3 is in agreement with the view that, over time, unique novel stimuli capture less attention. However, no significant amplitude reduction was found for the repeated administration of the identical set of novel stimuli. Cycowicz et al. (1996) also repeatedly presented stimuli from the same set of environmental sounds, but repetition was within a stimulus sequence, i.e. within a few minutes, whereas in the present study, a 7 days interval was employed. Although it is always a quandary to interpret the null hypothesis, this result points toward the view that stimulus familiarity not necessarily affects the novelty-P3 amplitude. Studies employing a systematic manipulation of the retest interval for the repeated presentation of novel stimuli may reveal in greater detail the temporal characteristics of habituation and dis-habituation of the novelty-P3.

In our study, target-P3 was not affected by block repetition within the two sessions, but was significantly reduced for the second as compared to the first recording session. A within session target-P3 amplitude reduction has been reported by Carrillode-la-Pena and Garcia-Larrea (1999), but only when the subjects had to perform a complex cognitive task between blocks. Other studies that reported habituation of target-P3 in an auditory oddball presented more target stimuli (Ivey and Schmidt, 1993), employed more experimental blocks (Pan et al., 2000; Romero and Polich, 1996), or did not find significant habituation until the last block (Pan et al., 2000). In addition, these studies performed a conventional two class auditory oddball, whereas a three class novelty oddball was used in the present study. Since we did not include a two class oddball, it remains an open question as to whether the additional presentation of task-irrelevant environmental sounds interacts with repetition effects on the target-P3.

Another limitation of the present study is that target-P3 session effects could not be paralleled by behavioral data. We failed to observe a significantly improved target detection from session 1 to session 2. This was possibly due to a ceiling effect, since the task difficulty employed was too low to reflect performance alterations between session 1 and session 2. Future work may consider a more difficult oddball task to address the question of improved performance, adjusted attentional demands, and its relation to the target-P3 amplitude. Additionally, the assessment of other behavioral variables like reaction times might be needed.

However, combining our results and those of the above-mentioned reports, it is likely that targetP3 alterations in the auditory novelty oddball reflect a different amount of cognitive resources that are allocated to perform the task. From this perspective, the novelty-P3 reflects an automatic, bottom-up driven aspect of attention, whereas the target-P3 is related to voluntary, top-down controlled attentional processes.

\section{Acknowledgments}

We are grateful to M. Fabiani for providing us with the novel stimuli. We also acknowledge the help of O. Haumann in data acquisition and thank M. Grigutsch (Max Planck Institute of Cognitive Neuroscience, Leipzig, Germany) for software support.

\section{References}

Beauducel, A., Debener, S., Brocke, B., Kayser, J., 2000. On the reliability of augmenting/reducing. Peak amplitudes and principal components analysis of auditory evoked potentials. J. Psychophysiol. 14, 226-240.

Carrillo-de-la-Pena, M.T., Garcia-Larrea, L., 1999. On the validity of interblock averaging of P300 clinical settings. Int. J. Psychophysiol. 34, 103-112.

Courchesne, E., Hillyard, S.A., Galambos, R., 1975. Stimulus novelty, task relevance, and the visual evoked potential in man. Electroencephalogr. Clin. Neurophysiol. 39, 131-141.

Cycowicz, Y.M., Friedman, D., Rothstein, M., 1996. A developmental study of the effect of temporal order on the ERPs elicited by novel environmental sounds. Electroencephalogr. Clin. Neurophysiol. 103, 304-318.

Daffner, K.R., Mesulam, M.M., Scinto, L.F.M., et al., 2000. The central role of the prefrontal cortex in directing attention to novel events. Brain 123, 927-939.

Debener, S., Beauducel, A., Nessler, D., Brocke, B., Heilemann, H., Kayser, J., 2000. Is resting anterior EEG alpha asymmetry a trait marker for depression? Neuropsychobiology 41, 31-37.

Donchin, E., Spencer, K.M., Dien, J., 1997. The varieties of deviant experience: ERP manifestations of deviance processors. In: van Boxtel, G.J.M., Bocker, K.B.E. (Eds.), Brain and Behaviour: Past, Present, and Future. University Press, Tilburg, The Netherlands, pp. 67-91. 
Fabiani, M., Friedman, D., 1995. Changes in brain activity patterns and aging: the novelty oddball. Psychophysiology 32, 579-594.

Friedman, D., Simpson, G., 1994. Amplitude and scalp distribution of target and novel events: effects of temporal order in young, middle-aged and older adults. Cogn. Brain Res. 2, 49-63.

Friedman, D., Cycowicz, Y.M., Gatea, H., 2001. The novelty P3: an event-related brain potential (ERP) sign of the brain's evaluation of novelty. Neurosci. Biobehav. Rev. 25, 355-373.

Herrmann, C.S., Knight, R.T., 2001. Mechanisms of human attention: event-related potentials and oscillations. Neurosci. Biobehav. Rev. 25, 465-476.

Humphreys, G.W., Duncan, J., Treisman, A., 1999. Attention, Space, and Action. Studies in Cognitive Neuroscience. first ed.. University Press, Oxford.

Ivey, R.G., Schmidt, H.B., 1993. P300 response: habituation. J. Am. Acad. Audiol. 4, 182-188.

Kayser, J., Tenke, C.E., Bhattacharya, N., Stuart, B.K., Hudson, J., Bruder, G.E., 2000. Direct comparison of geodesic sensor net (128-channel) and conventional (30-channel) ERPs in tonal and phonetic oddball tasks. Psychophysiology 37, S17.

Kinoshita, S., Inoue, M., Maeda, H., Nakamura, J., Morita, K., 1996. Long-term patterns of change in ERPs across repeated measurements. Physiol. Behav. 60, 1087-1092.

Knight, R.T., 1984. Decreased response to novel stimuli after prefrontal lesions in man. Electroencephalogr. Clin. Neurophysiol. 59, 9-20.

Lammers, W.J., Badia, P., 1989. Habituation of P300 to target stimuli. Physiol. Behav. 45, 595-601.
Luu, P., Ferree, T., 2000. Determination of the Geodesic Sensor Nets' Average Electrode Positions and their 10-10 International Equivalents. Electrical Geodesics, Inc, Eugene, Oregon, pp. 1-11.

Nunez, P.L., Silberstein, R.B., 2000. On the relationship of synaptic activity to macroscopic measurements: does coregistration of EEG with fMRI make sense? Brain Topogr. 13, 79-96.

Oken, B.S., Chiappa, K.H., 1986. Statistical issues concerning computerized analysis of brainwave topography. Annals Neurol. 19, 493-494.

Opitz, B., Mecklinger, A., Frederici, A.D., von Cramon, D.Y., 1999. The functional neuroanatomy of novelty processing: integrating ERP and fMRI results. Cereb. Cortex 9, 379-391.

Pan, J., Takeshita, T., Morimoto, K., 2000. P300 habituation from auditory single-stimulus and oddball paradigms. Int. J. Psychophysiol. 37, 149-153.

Polich, J., McIsaac, H.K., 1994. Comparison of auditory P300 habituation from active and passive conditions. Int. J. Psychophysiol. 17, 25-34.

Ravden, D., Polich, J., 1998. Habituation of P300 from visual stimuli. Int. J. Psychophysiol. 30, 359-365.

Romero, R., Polich, J., 1996. P3(00) habituation from auditory and visual stimuli. Physiol. Behav. 59, 517-522.

Simons, R.F., Graham, F.K., Miles, M.A., Chen, X., 2001. On the relationship of P3a and the novelty-P3. Biol. Psychol. 56, 207-218.

Sokolov, E.N., 1963. Perception and the Conditioned Reflex. Pergamon Press, Oxford, UK.

Tucker, D.M., 1993. Spatial sampling of head electrical fields: the geodesic sensor net. Electroencephalogr. Clin. Neurophysiol. 96, 538-545. 